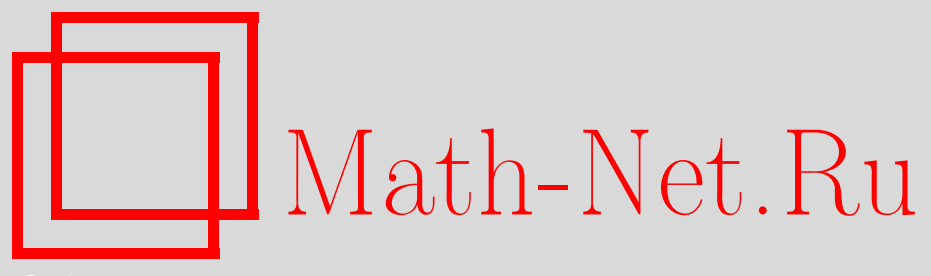

А. А. Приходько, Эргодические автоморфизмы с простым спектром и свойством быстрого убывания корреляций, Матем. заметки, 2013, том 94, выпуск 6, 949-954

DOI: https://doi.org/10.4213/mzm10144

Использование Общероссийского математического портала Math-Net.Ru подразумевает, что вы прочитали и согласны с пользовательским соглашением http://www.mathnet.ru/rus/agreement

Параметры загрузки:

IP : 3.89 .197 .203

26 апреля 2023 г., 17:55:54

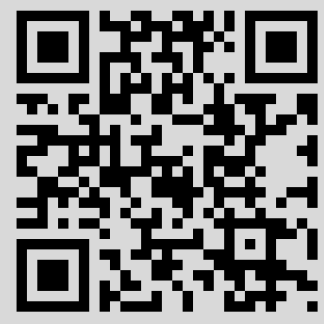




\section{Эргодические автоморфизмы с простым спектром и свойством быстрого убывания корреляций}

\section{А. А. Приходько}

В настоящей заметке мы докажем существование обратимых сохраняющих меру преобразований $T$, действующих на вероятностном пространстве Лебега $(X, \mathscr{A}, \mu)$, имеющих однократный (простой) спектр и допускающих для всюду плотного семейства функций $f \in L^{2}(X, \mathscr{A}, \mu)$ следующую оценку скорости убывания корреляций:

$$
\left\langle f\left(T^{k} x\right), f(x)\right\rangle=O\left(|k|^{-1 / 2+\varepsilon}\right) \quad \text { для всех } \varepsilon>0 .
$$

Учитывая тождество $\left\langle f\left(T^{k} x\right), f(x)\right\rangle=\widehat{\sigma}_{f}(k)$, где $\sigma_{f}$ - спектральная мера, отвечающая функции $f$, интересно отметить, что указанная скорость убывания коэффициентов Фурье $\widehat{\sigma}_{f}(k)$ является предельной возможной для сингулярных борелевских мер на $[0,1]$.

В эргодической теории преобразований с инвариантной мерой хорошо известна следующая проблема, поставленная Банахом: существует ли преобразование с инвариантной мерой, обладающее однократным лебеговским спектром? В книге Улама [1; гл. VI, $\S 6]$ эта проблема приводится в следующей формулировке: существует ли функиия $f \in$ $L^{2}(X, \mathscr{A}, \mu)$ и обратимое сохраняющее меру преобразование $T: X \rightarrow X$ такие, что последовательность функиий $\left\{f\left(T^{k} x\right): k \in \mathbb{Z}\right\}$ является полным оргональным семейством в гильбертовом пространстве $L^{2}(X, \mathscr{A}, \mu)$ ?

Легко привести пример динамической системы с таким свойством на пространстве с бесконечной мерой. Действительно, возьмем в качестве фазового пространства $X$ множество целых чисел $\mathbb{Z}$ со стандартной считающей мерой $\nu(\{j\}) \equiv 1$ и определим $T: j \mapsto j+1$. Тогда, очевидно, функции $\boldsymbol{\delta}_{k}=\boldsymbol{\delta}_{0} \circ T^{k}$ образуют базис в $L^{2}(\mathbb{Z}, \nu)$, где $\boldsymbol{\delta}_{k}(j)=1$ при $j=k$ и $\boldsymbol{\delta}_{0}(j)=0$ иначе.

Для пространств с конечной мерой вопрос Банаха открыт.

Кириллов [2] рассматривал следующее естественное обобщение вопроса Банаха: для каких топологических абелевых групп $G$ существует непрерывное действие группы $G$ с конечной инвариантной мерой, обладающее простым или конечнократным спектром, причем мера максимального спектрального типа эквивалентна мере Хаара на дуальной группе $\widehat{G}$ ?

В рамках заметки мы сконцентрируем наше внимание на случае $G=\mathbb{Z}$. Обзор основных конструкций и результатов спектральной теории динамических систем читатель может найти в [3] и [4].

Проблемы Банаха и Кириллова связаны с исследованием вопроса о скорости убывания на бесконечности корреляционных функций

$$
R_{f}(k)=\left\langle f\left(T^{k} x\right), f(x)\right\rangle, \quad f \in L^{2}(X, \mathscr{A}, \mu) .
$$

Дуальная группа $\widehat{\mathbb{Z}}$ изоморфна единичной окружности $S^{1}=\{z:|z|=1\}$ на комплексной плоскости, наделенной операцией умножения: характеры на группе $\mathbb{Z}$ имеют вид $\chi_{z}(k)=z^{k}$, где $z \in S^{1}$. Коэффициенты Фурье меры $\sigma$ на $S^{1}$ определяются равенством

$$
\widehat{\sigma}(k)=\int_{S^{1}} z^{k} d \sigma, \quad k \in \mathbb{Z} .
$$

ОПРЕДЕЛЕНИЕ 1. Обозначим символом $\kappa(\sigma)$ точную нижнюю грань значений $\alpha \in \mathbb{R}$, для которых выполнено: $\widehat{\sigma}(k)=O\left(|k|^{\alpha+\varepsilon}\right)$ при любом $\varepsilon>0$.

Поскольку группа $S^{1}$ компактна, любая борелевская мера, удовлетворяющая условию $\widehat{\sigma}(k)=O\left(|k|^{-1 / 2-c}\right)$ при некотором $c>0$, является абсолютно непрерывной относитель-

Работа выполнена при поддержке Российского фонда фундаментальных исследований (грант № 11-01-00759-а) и программы "Ведущие научные школы" (грант № НШ-5998.2012.1).

DOI: $10.4213 / \mathrm{mzm} 10144$ 
но нормированной меры Лебега $\lambda$ на $S^{1}$, т.е. $\sigma=p(x) \lambda$ для некоторой функции $p(x) \in$ $L^{1}\left(S^{1}, \lambda\right)$. Таким образом, любая мера на $[0,1]$ со свойством $\kappa(\sigma)<-1 / 2$ является абсолютно непрерывной.

Изучая спектральные свойства динамических систем, как правило, мы ограничиваемся рассмотрением функций с нулевым средним. Объясняется это тем, что константы всегда инвариантны относительно преобразования $\widehat{T}: f(x) \mapsto f(T x)$. Положим

$$
H^{\circ}=\left\{f \in L^{2}(X, \mathscr{A}, \mu): \int f d \mu=0\right\} .
$$

Для заданной функции $f \in H^{\circ}$ рассмотрим последовательность корреляций

$$
R_{f}(k)=\left\langle f\left(T^{k} x\right), f(x)\right\rangle
$$

и определим спектральную меру $\sigma_{f}$ равенством $\widehat{\sigma}_{f}(k)=R_{f}(k)$. Если неравенство $\kappa\left(\sigma_{f}\right)<$ $-1 / 2$ выполнено для плотного семейства функций $f$, то спектр преобразования $T$ абсолютно непрерывен.

Основным результатом работы является следующая теорема, которая утверждает, что граничное значение $\kappa\left(\sigma_{f}\right)=-1 / 2$ достижимо в классе эргодических преобразований с простым спектром.

Теорема 2. Существует обратимое сохраняющее меру преобразование $T$ пространства Лебега $L^{2}(X, \mathscr{A}, \mu)$ с простым спектром такое, что для плотного множества функиий $f$ верно неравенство $\kappa\left(\sigma_{f}\right) \leqslant-1 / 2$.

Идеи исследуемых нами конструкций восходят к работам по теории аппроксимации динамических систем, основы которой заложены в работах Катка, Стёпина и Оселедца (см. [5], [6]). Предложенный в данной работе класс динамических систем включает в себя класс автоморфизмов Орнстейна [7], которые в свою очередь являются важным примером систем ранга 1 (см. также [8]). Автоморфизм ранга 1 определяется как сохраняющее меру обратимое преобразование пространства Лебега $(X, \mathscr{A}, \mu)$, допускающее аппроксимацию последовательностью разбиений, связанных с башнями Рохлина,

$$
\xi_{n}=\left\{B_{n} T B_{n}, T^{2} B_{n}, \ldots, T^{h_{n}-1} B_{n}, E_{n}\right\}, \quad B_{n} \in \mathscr{A},
$$

где $\mu\left(E_{n}\right) \rightarrow \infty$. Известно, что системы ранга 1 обладают простым спектром [4]. Бурген показал [9], что спектр почти всех автоморфизмов Орнстейна сингулярен. Открыт вопрос о том, существует ли автоформизм ранга 1 , имеющий абсолютно непрерывную компоненту в спектре?

Рассмотренный в данной работе тип аппроксимации, обобщающий понятие аппроксимации ранга 1, позволяет доказать существование автоморфизмов со скоростью убывания корреляций $\left\langle T^{t} f, f\right\rangle=O\left(|t|^{-1 / 2+\varepsilon}\right)$, максимальной возможной для сингулярных мер на единичной окружности. В частности, $\sigma_{T} * \sigma_{T} \ll \lambda$, где $\sigma_{T}$ - мера максимального спектрального типа автоморфизма $T$ и $\lambda$ - мера Лебега. Одновременно сохраняется свойство простоты спектра. Рыжиков [10] доказал, что такая скорость убывания корреляций достижима в классе систем ранга 1, но на пространстве с бесконечной мерой. Вопрос о том, могут ли подобные эффекты наблюдаться для систем ранга 1 на пространстве с конечной мерой, пока остается открытым.

Пусть $[\mathscr{F}]$ - замыкание множества $\mathscr{F}$. Определим показатель Салема

$$
\kappa(T)=\inf _{[\mathscr{F}]=H^{\circ}} \sup _{f \in \mathscr{F}} \kappa\left(\sigma_{f}\right)
$$

и заметим, что теорема 2 утверждает существование автоморфизма $T$ с простым спектром такого, что $\kappa(T) \leqslant-1 / 2$. Если $\kappa(T)<-1 / 2$, то спектр преобразования $T$ абсолютно непрерывен. Отметим также, что $\kappa(T)=-\infty$ для любого $T$ с лебеговским спектром. Действительно, если унитарный оператор, связанный с автоморфизмом $T$, имеет лебеговскую компоненту в некотором подпространстве $H_{1} \subset H^{\circ}$, то существует элемент $e_{0} \in H_{1}$ такой, что $e_{k} \perp e_{j}$ при $k \neq j$, где $e_{k}(x)=e_{0}\left(T^{k} x\right)$. Очевидно, конечные линейные комбинации 
$g=\sum_{k=k_{1}}^{k_{2}} c_{k} e_{k}$ элементов $\left\{e_{k}\right\}$ плотны в $H_{1}$ и при этом для каждой такой функцции $g$ имеем $\left\langle g\left(T^{j} x\right), g(x)\right\rangle=0$, как только $|j|>k_{2}-k_{1}$. Это означает, что $\kappa\left(\left.\widehat{T}\right|_{H_{1}}\right)=-\infty$. Таким образом, если $H_{1}=H^{\circ}$, то $\kappa(T)=-\infty$.

Теорема 3. Пусть $T$ - автоморфизм пространства Лебега, обладающий свойством $\kappa\left(\sigma_{f}\right)=-1 / 2$, и пустъ $\sigma$ - мера максимального спектрального типа автоморфизма $T$. Тогда $\sigma * \sigma \ll \lambda u$, кроме того, либо спектр $T$ содержит абсолютно непрерывную компоненту, либо спектр $T$ является чисто сингулярным, $\kappa\left(\sigma_{f}\right) \geqslant-1 / 2$ и для плотного семейства функиий $f$ выполнено $\kappa\left(\sigma_{f}\right)=-1 / 2$.

ДоказАтельство. В самом деле, если существует функция $f^{*}$ такая, что $\kappa\left(\sigma_{f} *\right)<$ $-1 / 2$, то в силу сделанного выше замечания спектральная мера $\sigma_{f *}$ содержит абсолютно непрерывную компоненту $\eta$, а значит, $\eta \ll \sigma$. Далее будем предполагать, что $\kappa\left(\sigma_{f}\right) \geqslant-1 / 2$ для всех $f$. По-прежнему существует потенциальная возможность наличия абсолютно непрерывной компоненты. Таким образом, если исключить первый пункт альтернативы, то для любого элемента $f$ мера $\sigma_{f}$ сингулярна и спектральный тип $\sigma$ преобразования также сингулярен, причем $\kappa\left(\sigma_{f}\right) \geqslant-1 / 2$. Кроме того, в условиях утверждения всегда можно найти плотное семейство функций $\mathscr{F}$, демонстрирующих быстрое убывание корреляций: $\kappa\left(\sigma_{f}\right)=-1 / 2$ для $f \in \mathscr{F}$.

Сингулярные борелевские меры такого типа, которые мы будем называть мерами Салема-Шефера, впервые исследовались в работах Шефера, Салема, Зигмунда, Ивашева-Мусатова и др. (см., например, [11]-[13]).

Цель настоящей работы - показать, как строятся динамические системы, обладающие свойствами, указанными в теореме 2. Мы рассмотрим две эквивалентных конструкции, одна из которых использует язык символической динамики, а другая опирается на геометрическую конструкцию преобразования. Затем мы изложим схему доказательства теоремы 2.

Символическая конструкция. Пусть $\mathbb{A}$ - конечный алфавит и $w_{0}$ - некоторое начальное слово в алфавите $\mathbb{A}$, содержащее хотя бы две различные буквы. Обозначим символом $\rho_{\alpha}$ преобразование ииклического сдвига:

$$
\rho_{1}(a u)=u a, \quad \rho_{\alpha}(u)=\rho_{1}^{\alpha}(u), \quad a \in \mathbb{A}-\text { буква, } \quad u-\text { слово. }
$$

Построим последовательность слов $w_{n}$ по следующему правилу:

$$
w_{n+1}=\rho_{\alpha_{n, 0}}\left(w_{n}\right) \rho_{\alpha_{n, 1}}\left(w_{n}\right) \ldots \rho_{\alpha_{n, q_{n}-1}}\left(w_{n}\right) \text {. }
$$

Последовательности $q_{n} \in \mathbb{N}$ и $\alpha_{n, j} \in \mathbb{Z} / h_{n} \mathbb{Z}, h_{n}=\left|w_{n}\right|$, являются параметрами конструкции. Здесь $|w|$ - длина слова $w$. Не ограничивая общности, можно считать, что к первому вхождению слова $w_{n}$ в $w_{n+1}$ циклический сдвиг не применяется. Тогда каждое $w_{n}$ является префиксом следующего в последовательности слова $w_{n+1}$ и корректно определено одностороннее бесконечное слово $w_{\infty}$, расширяющее вправо каждое из слов $w_{n}$.

Определим далее, следуя стандартной в топологической динамике процедуре, компактное подмножество $K \subset \mathbb{A}^{\mathbb{Z}}$ множества всех двусторонних бесконечных последовательностей $\mathbb{A}^{\mathbb{Z}}$, являющееся замыканием в слабой топологии сдвигов слова $w_{\infty}$. На множестве $K$ действует естественное преобразование сдвига $T:\left(x_{j}\right) \mapsto\left(x_{j+1}\right)$. Снабдим множество $K$ борелевской мерой $\mu$, которая в терминах символической динамики задается правилом: вероятность $\mu(u)$ слова $u$ равна асимптотической частоте наблюдения $u$ в качестве подслова в $w_{\infty}$. Мера $\mu$ сохраняется преобразованием $T$.

Конструкция и основные эргодические свойства системы $(T, K, \mu)$ подробно исследованы в [14]. Топологическая система $(T, K)$, а также положенное в основу определения бесконечное слово $w_{\infty}$ представляют интерес с точки зрения теории сложности динамических систем [15]. Символическая конструкция, аналогичная одному шагу в (1), но с бесконечной последовательностью циклических сдвигов копий слова $w_{n}$, в теории алгоритмов носит название вальса бесконечного порядка [16]. Рассмотренная нами конструкция также 
обобщает некоторые известные эргодические системы. А именно, полагая $h_{1}=2, q_{n} \equiv 2$, $\rho_{n, 0}=0, \rho_{n, 1}=h_{n} / 2$, мы приходим к сохраняющему меру преобразованию, известному как автоморфизм Морса (см., например, [17]). Кроме того, построенная система допускает реализацию в виде адического преобразования.

Алгебраическая конструкция. Пусть $h_{n}$ - некоторая поледовательность натуральных чисел, удовлетворяющая соотношению $h_{n+1}=q_{n} h_{n}, q_{n} \in \mathbb{N}, q_{n} \neq 1$. Рассмотрим последовательность вложенных решеток $\Gamma_{n}=h_{n} \mathbb{Z}, \Gamma_{n+1} \subset \Gamma_{n}$, и соответствующую последовательность (конечных) однородных пространств $M_{n}=\mathbb{Z} / \Gamma_{n}=\mathbb{Z}_{h_{n}}$. Заметим, что пространства $M_{n}$ связаны естественной проекцией $\pi_{n}: y+\Gamma_{n+1} \mapsto y+\Gamma_{n}$. Мы рассмотрим более широкий класс проекций $\phi_{n}: M_{n+1} \rightarrow M_{n}$,

$$
\phi_{n}: j h_{n}+k \mapsto \alpha_{n, j}+k \quad\left(\bmod h_{n}\right), \quad 0 \leqslant k<h_{n}, \quad j=0,1, \ldots, q_{n}-1 .
$$

Отображения $\phi_{n}$ сохраняют нормированные меры Хаара $\mu_{n}$ на $M_{n}$. Определим фазовое пространство $X$ динамической системы как обратный предел пространств $\left(M_{n}, \mu_{n}\right)$, а именно, положим

$$
X=\left\{x=\left(x_{1}, x_{2}, \ldots, x_{n}, \ldots\right): \phi_{n}\left(x_{n+1}\right)=x_{n}\right\} .
$$

Меры $\mu_{n}$ продолжаются до борелевской меры $\mu$ на $X$. Наконец, зададим действие преобразования $T$ пространстве $X$ по следующей схеме. Заметим, что отображение $\phi_{n}$ почти коммутирует с преобразованиями сдвига на $M_{n}$,

$$
\mu\left\{x: \phi_{n}\left(S_{n+1}\left(x_{n+1}\right)\right) \neq S_{n}\left(\phi_{n}\left(x_{n+1}\right)\right)\right\} \leqslant h_{n}^{-1} .
$$

Используя это наблюдение и применяя лемму Бореля-Кантелли, легко проверить, что для $\mu$-почти всех точек $x \in X$ указанное соотношение коммутирования выполнено для всех $n \geqslant n_{1}(x)$. Определим теперь действие преобразования $T$ на точку $x$ соотношениями

$$
(T x)_{n}=x_{n}+1, \quad n \geqslant n_{1}(x), \quad \text { и } \quad(T x)_{n}=\phi_{n}\left(T x_{n+1}\right), \quad n<n_{1}(x) .
$$

Верна следующая лемма (см. [14]).

Лемма 4. Преобразование Т является обратимым сохраняющим меру преобразованием (автоморфизмом) пространства Лебега $(X, \mathscr{A}, \mu)$.

Эквивалентность двух рассмотренных конструкций устанавливается при помощи процедуры кодирования орбит преобразования $T$ словами $w_{n}$, которые мы интерпретируем как функции $M_{n} \rightarrow \mathbb{A}$.

Конструкция 5. Для доказательства теоремы 2 мы рассмотрим стохастическое семейство динамических систем указанного вида, зависящее от набора случайных параметров, и покажем, что построенные таким образом автоморфизмы обладают свойствами, заявленными в теореме 2, почти наверное относительно вероятности на пространстве параметров.

Теорема 6. Для некоторой возрастающей последовательности $q_{n} \in \mathbb{N}$ и параметров $\alpha_{n, j}$, распределенных независимо и равномерно на $M_{n}$, автоморфизм $T$, построенный в соответствии с рассмотренной конструкиией, почти наверное имеет простой спектр и скорость убывания корреляиий

$$
\left\langle f\left(T^{t} x\right), f(x)\right\rangle=O\left(|t|^{-1 / 2+\varepsilon}\right)
$$

при всех $\varepsilon>0$ для плотного семейства элементов $f \in L^{2}(X, \mathscr{A}, \mu)$.

СХема ДОКАЗАТЕЛЬСТвА теоремы 6. Оценим вначале скорость убывания корреляций. Для этого достаточно исследовать корреляционные функции, отвечающие плотному в $L^{2}(X, \mu)$ семейству цилиндрических функций вида $f(x)=f_{n_{0}}\left(x_{n_{0}}\right)$, где $f_{n_{0}}: M_{n_{0}} \rightarrow \mathbb{C}$ 
(см. [14]). Функцию $f(x)$ можно представить для любого $n>n_{0}$ в виде $f(x)=f_{n}\left(x_{n}\right)$, где $f_{n+1}\left(x_{n+1}\right)=f_{n}\left(\phi_{n}\left(x_{n+1}\right)\right)$. Определим функции периодических корреляций

$$
R_{f}^{\circ}(t)=\int_{M_{n}} f_{n}(j+t) \overline{f_{n}(j)} d \mu_{n}(j) .
$$

Не ограничивая общности, рассмотрим наиболее нетривиальный случай $t=s h_{n}, s \neq 0$. Для такого специального значения аргумента $t$ верна рекуррентная формула

$$
R_{n+1}^{\circ}(t)=\frac{1}{q_{n}} \sum_{k=0}^{a_{n}-1} R_{n}^{\circ}\left(\alpha_{n, k+s}-\alpha_{n, k}\right) .
$$

Применяя оператор математического ожидания относительно вероятности на пространстве параметров, имеем: $\mathrm{E} R_{n+1}^{\circ}(t)=\mathrm{E} R_{n}^{\circ}\left(\alpha_{n, k+s}-\alpha_{n, k}\right)=0$ и

$$
\mathrm{E}\left|R_{n+1}^{\circ}(t)\right|^{2}=\frac{1}{q_{n}^{2}} \mathrm{E} \sum_{k, \ell=0}^{q_{n}-1} R_{n}^{\circ}\left(\alpha_{n, k+s}-\alpha_{n, k}\right) \overline{R_{n}^{\circ}\left(\alpha_{n, \ell}-\alpha_{n, \ell+s}\right)},
$$

откуда следует, что $\mathrm{E}\left|R_{n+1}^{\circ}(t)\right|^{2}=h_{n+1}^{-1} \mathrm{E}\left\|R_{n}^{\circ}\right\|^{2}$, где $\left\|R_{n}^{\circ}\right\|^{2}-$ стандартная норма в $L^{2}(\mathbb{Z})$. Таким образом, мы видим, что

$$
\mathrm{E}\left\|R_{n+1}^{\circ}\right\|^{2} \leqslant 2 \cdot \mathrm{E}\left\|R_{n}^{\circ}\right\|^{2},
$$

и, применяя ряд дополнительных оценок, видим, что $R_{n+1}^{\circ}(t)=O\left(|t|^{-1 / 2+\varepsilon}\right)$ при любом $\varepsilon>0$, и второе утверждение теоремы доказано.

Доказательство первой части теоремы 6 опирается на следующую лемму.

Лемма 7 [18]. Пусть $U$ - унитарный оператор в сепарабельном гильбертовом пространстве $H, \sigma$ - мера максимального спектрального типа и $\mathcal{M}(z)$ - функиия кратности оператора $U$. Если $\mathcal{M}(z) \geqslant m$ на множестве положительной $\sigma$-меры, то найдутся $m$ ортогональных элементов единичной длины $f_{1}, \ldots, f_{m}$ такие, что для произвольного ииклического пространства $Z \subset H$ (относительно $U$ ) и для любых $m$ элементов $g_{1}, \ldots, g_{m} \in Z$ равной длины $\left\|g_{i}\right\|=а$ выполнено неравенство

$$
\sum_{i=1}^{m}\left\|f_{i}-g_{i}\right\|^{2} \geqslant m\left(1+a^{2}-\frac{2 a}{\sqrt{m}}\right) .
$$

Пусть $\xi_{n}$ - разбиение пространства $X$ на множества $A_{n, y}=\left\{x: x_{n}=y\right\}$, где $y \in M_{n}$. Идея доказательства простоты спектра заключается в аппроксимации на части фазового пространства любой $\xi_{n}$-измеримой функции итерациями $\left\{T^{k} F_{n}\right\}$, где $F_{n}(x)$ - индикатор множества $A_{n, 0}$. Подробное рассуждение приведено в [14]. Данный случай применения леммы 7 примечателен тем, что удается оценить кратность спектра при отсутствии циклической аппроксимации или конечного ранга.

Важной особенностью данного метода оценивания скорости убывания коэффициентов Фурье $\widehat{\sigma}_{f}(n)$ является использование исключительно оценок для моментов корреляционных функций. В самом деле, полученная скорость убывания корреляций является простым следствием конструкции преобразования. В то же время, полное исследование распределений случайных $R_{n}^{\circ}(t)$, равно как изучение структуры спектральных мер $\sigma_{f}$, представляет собой крайне нетривиальную задачу. Отметим, что указанная скорость убывания корреляций $O\left(|t|^{-1 / 2+\varepsilon}\right)$ может наблюдаться как для сингулярных, так и для абсолютно непрерывных распределений.

Дальнейшее изучение обнаруженного явления, состоящего в достижении предельной скорости убывания корреляций для систем с сингулярным спектром, приводит к гипотезе о том, что в рассмотренном классе автоморфизмов данная оценка является неулучшаемой и скорость убывания корреляций, вычисленная для семейства стандартных цилиндрических функций, не позволяет определить, является ли мера максимального спектрального типа чисто сингулярной или содержит абсолютно непрерывную компоненту. 
ОПРедЕЛЕНиЕ 8. Автоморфизм пространства Лебега допускает аппроксимацию тu$n a \mathcal{I}$, если для любого $\varepsilon>0$ найдется подмножество $\Omega_{\varepsilon} \subset X$ меры $1-\varepsilon$ и слово $W_{\varepsilon}$ такое, что для всех $x \in \Omega_{\varepsilon}$ орбита точки $x$ с точностью до $\varepsilon$ накрывается последовательностью дизъюнктных подслов $W_{j}$, которые $\varepsilon$-близки к словам вида $\rho_{\alpha}\left(W_{\varepsilon}\right)$.

Данное свойство является метрическим инвариантом. Конечно, рассмотренная в данной работе явная конструкция порождает преобразования, допускающие аппроксимацию типа $\mathcal{I}$. При $\alpha=0$ данное определение задает класс автоморфизмов ранга 1.

ГиПотезА 9. Пусть $T$ - автоморфизм, построенный в результате конструкции 5 и зависящий от случайного параметра. Для почти каждого $T$ выполнено следующее: для любого $f \in L^{2}(X, \mathscr{A}, \mu)$ имеет место оценка $\kappa\left(\sigma_{f}\right) \geqslant-1 / 2$.

ГипотезА 10. Предположим, что $T$ - автоморфизм, допускающий аппроксимацию типа $\mathcal{I}$, и $\xi_{n}-$ некоторая универсальная последовательность аппроксимирующих разбиений такая, что для любого конечного разбиения $\mathcal{P}$, индексированного символами алфавита $\mathbb{A}$, бесконечные $\mathcal{P}$-коды орбит асимптотически аппроксимируются с точностью до $\varepsilon_{n}$ словами $\rho_{\alpha}\left(W_{(n)}\right)$, где $W_{(n)}$ - слова, связанные с разбиениями $\xi_{n}$, и $\varepsilon_{n} \rightarrow 0$. Тогда

$$
\varliminf_{n \rightarrow \infty} \inf _{f \in L^{2}\left(\xi_{n}\right)} \kappa\left(\sigma_{f}\right) \geqslant-\frac{1}{2},
$$

где $L^{2}\left(\xi_{n}\right)$ - пространство квадратично суммируемых функций, измеримых относительно разбиения $\xi_{n}$.

Автор благодарен А. М. Вершику, В. В. Рыжикову, Ж.-П. Тувено, Э. Жанврез, М. Леманчику, Т. де ля Рю, К. Петерсону, Б. Вейсу, а также участникам семинара "Теория вероятностей и статистическая физика" на механико-математическом факультете МГУ им. М. В. Ломоносова за интерес к данному исследованию и полезные замечания.

\section{СПИСОК ЦИТИРОВАННОЙ ЛИТЕРАТУРЫ}

[1] С. Улам, Нерешенные математические задачи, Современные проблемы математики, Наука, М., 1964. [2] А.А. Кириллов, УМН, 22:5(137) (1967), 67-80. [3] A. Katok, J.-P. Thouvenot, Handbook of Dynamical Systems, Vol. 1B, Elsevier, Amsterdam, 2006. [4] M. Lemańczyk, Encyclopedia of Complexity and System Science, Springer-Verlag, Berlin, 2009, 8554-8575. [5] А. Б. Каток, А. М. Степин, УМН, 22:5 (1967), 81-106. [6] В. И. Оселедец, Матем. заметки, 5:3 (1969), 323-326. [7] D. S. Ornstein, Proceedings of the Sixth Berkeley Symposium on Mathematical Statistics and Probability. Vol. II. Probability Theory, Univ. California Press, Berkeley, CA, 1972, 347-356. [8] S. Ferenczi, Colloq. Math., 73:1 (1997), 35-65. [9] J. Bourgain, Israel J. Math., 84:1-2 (1993), 53-63. [10] V. V. Ryzhikov, On Mixing Rank One Infinite Transformations, 2011, arXiv: math.DS/1106.4655. [11] A.C. Schaeffer, Amer. J. Math., 61:4 (1939), 934-940. [12] R. Salem, Trans. Amer. Soc. Math., 53 (1943), 427-439. [13] О. С. Ивашев-Мусатов, Изв. АН СССР. Сер. матем., 20:2 (1956), 179-196. [14] A. A. Prikhod'ko, On Ergodic Properties of Iceberg Transformations. I: Approximation and Spectral Multiplicity, 2010, arXiv: math.DS/1008.4301. [15] A. A. Prikhod'ko, Lower Bounds for Symbolic Complexity of Iceberg Dynamical Systems, 2011, arXiv: math.DS/ 1201.5757. [16] Г.-Д. Эббинхауз, К. Якобс, Ф.-К. Ман, Машины Тьюринга и рекурсивные функиии, Популярная серия "Современная математика", Мир, М., 1972. [17] А. М. Вершик, Б. Соломяк, Теория представлений, динамические системы, комбинаторные методы. XVI, Зап. научн. сем. ПОМИ, 360, ПОМИ, СПб., 2008, 70-90. [18] И. П. Корнфельд, Я. Г. Синай, С. В. Фомин, Эргодическая теория, Наука, М., 1980.

\section{А. А. Приходько}

Поступило

Московский государственный

31.07 .2012

университет им. М. В. Ломоносова

E-mail: sasha.prihodko@gmail.com

Исправленный вариант

22.02 .2013 